\title{
Periglaziale Formung am Kendrick Peak in Nord-Arizona während der letzten Kaltzeit ${ }^{1}$
}

\author{
Dietrich Barsch, Geographisches Institut, Universität Basel \\ Randell G. Updike, Department of Geology, Arizona State University
}

\section{Einleitung}

Die letztkaltzeitliche (wisconsin-zeitliche) periglaziale Formung ist in den höher aufragenden Gebirgen des ariden und semiariden Südwestens der Vereinigten Staaten bisher noch wenig untersucht worden. ${ }^{2}$ Die Probleme der eiszeitlichen Vergletscherung und die Probleme der pluvialen Seen haben stärker im Vordergrund des Interesses gestanden. Dabei ist aber gerade die fossile periglaziale oder subnivale Höhenstufe in den Gebirgen der heutigen Trockengebiete viel ausgedehnter gewesen als die glaziale; sie bietet deshalb auch viel mehr Hinweise zur Rekonstruktion der letztkaltzeitlichen Umwelt in diesen schon recht südlich gelegenen Gebieten. In diesem Zusammenhang ist gerade die detaillierte Bestimmung der Untergrenze der wisconsin-zeitlichen subnivalen Höhenstufe in den Trockengebieten eine notwendige Voraussetzung für ein besseres Verständnis der dreidimensionalen Verschiebungen der geomorphologischen, der klimatischen und der vegetations-geographischen Zonen auf der Erde während der letzten Kaltzeit.

Um Hinweise auf die periglaziale Formung in dem noch wenig untersuchten Arizona zu bekommen, das immerhin mit $296000 \mathrm{~km}^{2}$ siebenmal größer ist als die Schweiz oder $20 \%$ mehr Fläche umfaßt als die Bundesrepublik Deutschland, haben wir das Gebiet von Kendrick Peak bearbeitet. Kendrick Peak war mit 3175 m Höhe in der letzten Kaltzeit nicht vergletschert, kann jedoch nur knapp unter der damaligen Schneegrenze gelegen haben, deren Höhe in den nur $10 \mathrm{~km}$ weiter östlich gelegenen San Francisco Mountains zu 3300 m (11000 ft) bestimmt wurde (Updike, 1969). Kendrick Peak dürfte also eine ausgedehnte subnivale Höhenstufe besessen haben. Aus diesem Grunde haben wir hier eine erste Untersuchung des fossilen periglazialen Formenschatzes angesetzt.

\section{Lage des Untersuchungsgebietes}

Kendrick Peak (vgl. Fig. 1) liegt im nördlichen Arizona etwas südlich des Grand Canyon auf $35^{\circ} 25^{\prime}$ nördlicher Breite und $115^{\circ} 50^{\prime}$ westlicher Länge. Das Berggebiet gehört zusammen mit den San Francisco Mountains zu den bedeutendsten Erhebungen nördlich der Mogollon Rim, der gro-
Ben Bruchstufe, welche Arizona von NW nach SE quert und welche die großen morpho-tektonischen Regionen des Südwestens der USA, die "Basinand-Range-Provinz" und die "Colorado-PlateauProvinz» voneinander trennt. Der Höhenunterschied an der Bruchstufe beträgt einige hundert Meter. So liegt das Coloradoplateau in der Umgebung von Flagstaff in etwas über 2000 m Höhe, die Beckenlandschaften südlich der Mogollon Rim meist nur auf 300 bis $1500 \mathrm{~m}$ Höhe. Entlang dieser Bruchstufe bilden vulkanische Erscheinungen tertiären und quartären Alters keine Seltenheit. Eines der bedeutendsten Vulkanfelder stellt das «San Francisco vulcanic field» dar, dessen höchster Gipfel, Humphreys Peak (3851 m), nur $10 \mathrm{~km}$ östlich von Kendrick Peak liegt. Dieses Feld ist bis in die jüngste Vergangenheit vulkanisch aktiv gewesen (Ausbruch des Sunset Crater östlich der San Francisco Peaks: etwa 1064 n. Chr. nach Colton 1967, p. 36).

Das isolierte Berggebiet von Kendrick Peak ist als Teil des Vulkanfeldes der San Francisco Peaks anzusehen. Es ist ganzseitig von größeren Ebenheiten umgeben, die Kendrick Peak mit einer Höhe von $3175 \mathrm{~m}$ um ungefähr $900 \mathrm{~m}$ überragt. Das Berggebiet umfaßt eine Fläche von etwa 47 km².

\section{Klima}

Im Berggebiet des Kendrick Peak sind bisher keine meteorologischen Messungen durchgeführt worden. Die nächste meteorologische Station liegt $28 \mathrm{~km}$ weiter südöstlich auf $2070 \mathrm{~m}$ Höhe $(6903 \mathrm{ft})$ in Flagstaff. Das Klima von Flagstaff (Tab. 1) zeichnet sich gegenüber dem wüstenhaften, heißen Klima der Beckenlandschaften des mittleren und südlichen Arizona (z. B. Phoenix) durch kühlere Temperaturen und größere Feuchtigkeit aus, wobei die Niederschläge im Winter an pazifische Luftmassen gebunden sind, die über die Sierra Nevada nach $\mathrm{E}$ vordringen. Die Niederschläge des Sommers (Juli, August) entstammen dagegen tropischen Luftmassen aus dem Gebiet des Golfes von Mexiko. Sie fallen meist nachmittags in Form von Wolkenbrüchen (Gewittern). Als Regel gilt (Green and Sellers 1964), daß etwa 75\% der winterlichen Niederschläge in Flagstaff als Schnee fallen. Flagstaff zeigt so ein deutliches Höhenklima im Bereich der rand- 


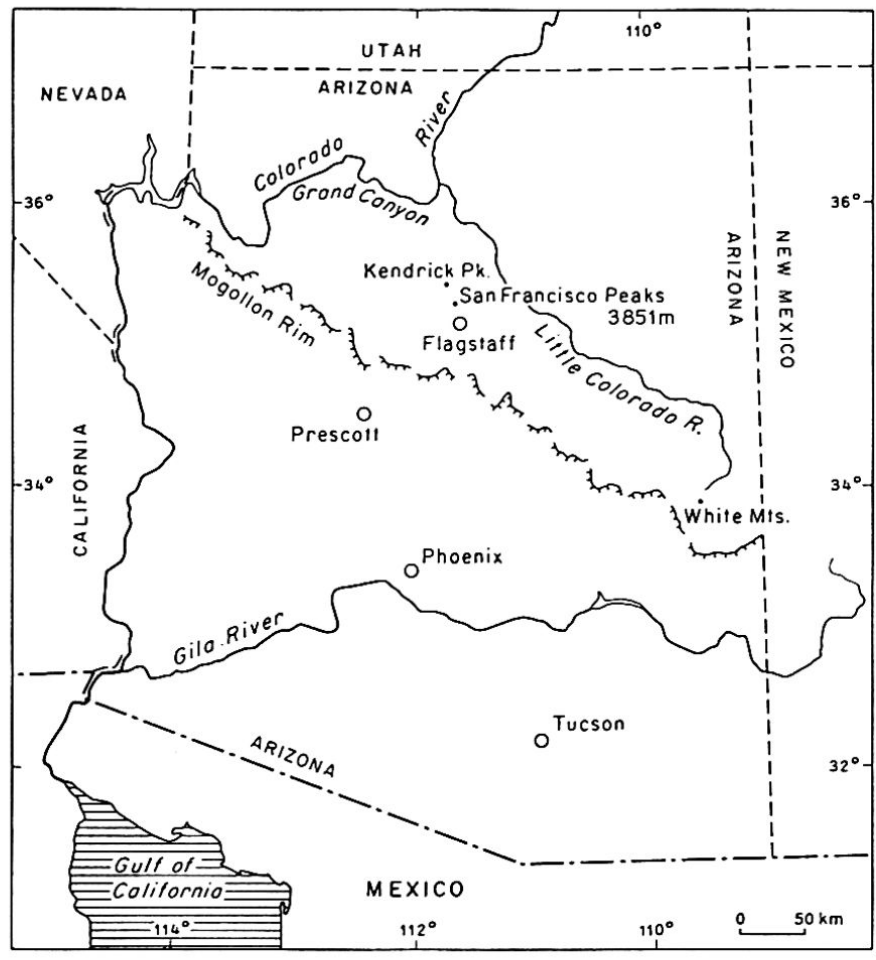

Figur 1. Übersichtsskizze. Index map

tropischen Wüsten, deren Klima bei Phoenix nach der Klimaklassifikation von Koeppen als BWh, nach der von Troll und Paffen (1964) als IV/5 zu bezeichnen ist.

Bei Annahme eines Temperaturgradienten von 0,5 bis $0,6^{\circ} \mathrm{C} / 100 \mathrm{~m}$ errechnet sich gegenwärtig für Kendrick Peak in $3000 \mathrm{~m}$ Höhe eine mittlere Jahrestemperatur von etwa $1,3-2,3^{\circ} \mathrm{C}$. Das Jahresmittel ist danach also noch deutlich positiv.

Sehr viel schwieriger ist eine Abschätzung der Niederschlagsmenge, die die Höhenregionen des Kendrick Peak gegenwärtig erhalten. Als Hinweis können nur die Werte dienen, die in den San Francisco Mountains gemessen wurden. Nach Colton (1958) beträgt die mittlere jährliche Niederschlagsmenge im nach E geöffneten Haupttal der San Francisco
Mountains, im sogenannten Interior Valley, in $3000 \mathrm{~m}$ Höhe rund $838 \mathrm{~mm}$ (33 in.), wobei in extremen Jahren Werte von 270 bzw. $1154 \mathrm{~mm}$ erreicht werden. Die Niederschläge nehmen in diesen isolierten Berggebieten mit der Höhe stark zu.

Im Winterhalbjahr, genauer von Ende Oktober bis Ende April, fallen die Niederschläge in der Höhe als Schnee. Der Schnee selbst bleibt in einzelnen Flecken im Nadelwald der Gipfelregion bis Ende Juni oder Anfang Juli liegen.

\section{Vegetation}

Die Tatsache, daß sich in Arizona mit den San Francisco Mountains ein Berggebiet aus den wintermilden Wüsten im Süden und den winterkalten Wüsten im Norden bis knapp 4000 m Höhe erhebt, hat schon frühzeitig die Aufmerksamkeit der Botaniker und Vegetationsgeographen auf die vertikale Gliederung der Vegetation gelenkt. Es ist deshalb kein Wunder, daß Merriam (1890) in dieser Region sein klassisches Konzept der Vegetationszonen für Nordamerika ( «life zones») entwickelt hat. Merriam versucht, mit seinem Modell die zonale und vertikale Abfolge der Vegetation zu beschreiben. Allerdings ist dieses Beschreibungsmodell heute bei Botanikern und Forstleuten nicht unbestritten. Es erfaßt zweifellos nicht alle durch edaphische und vegetationsgeschichtliche Einflüsse bedingten Feinheiten der Pflanzenverteilung, doch liegt ihm ein tatsächlich immer wieder zu beobachtender Zusammenhang zugrunde. Dadurch eignet es sich aber - trotz zahlreicher Einwände - für eine klimatische und vegetationsgeographische Kurzbeschreibung der einzelnen Höhenzonen, die für eine Parallelisierung mit rezenten oder fossilen geomorphologischen Höhenzonen auf alle Fälle genau genug ist.

Für Nordamerika unterscheidet Merriam die folgenden sechs Vegetationszonen (vgl. Fig. 2).

Tabelle 1. Klimadaten von Flagstaff und Phoenix, Arizona (nach Green und Sellers 1964)

\begin{tabular}{|c|c|c|c|c|c|c|c|c|c|c|c|c|c|c|c|}
\hline Station & Periode & & Jan. & Febr. & März & April & Mai & Juni & Juli & Aug. & Sept. & Okt. & Nov. & Dez. & al \\
\hline $\begin{array}{l}\text { Flagstaff } \\
(2070 \mathrm{~m})\end{array}$ & $\begin{array}{l}1899- \\
1951\end{array}$ & $\begin{array}{l}\mathrm{T} \text { in } \\
{ }^{\circ} \mathrm{C}\end{array}$ & $-2,4$ & $-0,7$ & 2,3 & 6,2 & 10,2 & 15,1 & 18,7 & 17,7 & 14,1 & 8,2 & 2,9 & $-1,3$ & \\
\hline & & & 49,5 & 50,3 & 48,5 & 33,0 & 17,3 & 12,2 & 72,6 & 72,6 & 45,4 & 38,1 & 31,0 & 44,2 & \\
\hline $\begin{array}{l}\text { hoenix } \\
325 \mathrm{~m})\end{array}$ & $\begin{array}{l}1896- \\
1957\end{array}$ & $\begin{array}{l}\mathrm{T} \text { in } \\
{ }^{\circ} \mathrm{C}\end{array}$ & 11,2 & 13,3 & 15,9 & 20,1 & 24,5 & 29,6 & 32,5 & 31,6 & 28,7 & 22,2 & 15,6 & 11,6 & \\
\hline & & $\begin{array}{l}\mathrm{N} \text { in } \\
\mathrm{nm}\end{array}$ & 20,8 & 19,6 & 17,3 & 10,7 & 3,05 & 1,78 & 26,9 & 26,9 & 20,8 & 11,2 & 15,7 & 20,1 & \\
\hline
\end{tabular}


Lower Sonoran life zone

Upper Sonoran life zone

Transition life zone

Canadian life zone

Hudsonian life zone

Arctic-Alpine life zone

In Nordarizona findet sich der Höhe wegen die unterste dieser Zonen, die Lower Sonoran life zone sowie ihre Charakterpflanze, der Kandelaberkaktus («saguaro», Cereus giganteus), nicht mehr. Am Kendrick Peak selbst treten nur drei, nämlich die Transition life zone, die Canadian life zone und die Hudsonian life zone auf, welche im folgenden etwas näher beschrieben werden sollen.

In unserem Berggebiet wird die Waldgrenze, die in den San Francisco Mountains heute bei 3400 $3600 \mathrm{~m}$ liegt, nicht erreicht. Die Gipfelregion und vor allem die hohen Nordhänge von Kendrick Peak sind deshalb durch fast reine Bestände der Engelmann spruce (Picea Engelmanni), die als dominierende Art der Hudsonian life zone anzusehen ist, gekennzeichnet. Beigemischt sind der Engelmann spruce vor allem Bristlecone pine (Pinus aristata), die in den San Francisco Mountains stellenweise die Waldgrenze bildet. Die Untergrenze der Hudsonian life zone dürfte je nach Exposition und Untergrund bei 2700 bis $2900 \mathrm{~m}$ liegen.

Darunter folgt der typische gemischte Nadelwald der Canadian life zone, der einen großen Teil des untersuchten Berggebietes bedeckt. Er besteht aus verschiedenen Nadelhölzern, vorherrschend jedoch aus Douglas fir (Pseudotsuga menziesi). Im oberen Teil dieser Zone sind der Douglas fir unter anderem Bristlecone pine und Engelmann spruce beigemischt; im unteren Teil die bekannte Ponderosa pine (Pinus ponderosa). Die Untergrenze der Canadian life zone dürfte bei 2450 bis $2600 \mathrm{~m}$ liegen. Darunter folgt der reine Kiefernwald aus Ponderosa pine, der die Fußregion und die Flächen um den Berg bedeckt, soweit es sich nicht um echte "parks» handelt, in denen meist wohl aus edaphischen Gründen natürlicherweise kein Wald, sondern Gras wächst. Solche offenen, waldfreien Stellen findet man auch in den höheren Teilen des Berges. Dort erreichen sie jedoch flächenmäßig nicht die Bedeutung, die sie in der Fußregion besitzen.

\section{Gesteinsbeschaffenheit}

Wie schon betont wurde, stellt das Berggebiet von Kendrick Peak einen Teil des San Francisco vulcanic field dar. Kendrick Peak selbst ist ein erloschener Vulkan, der in fünf eruptiven Perioden aufgebaut wurde (Robinson, 1913). Den einzelnen Lavaflüssen entstammen in der Reihenfolge ihrer

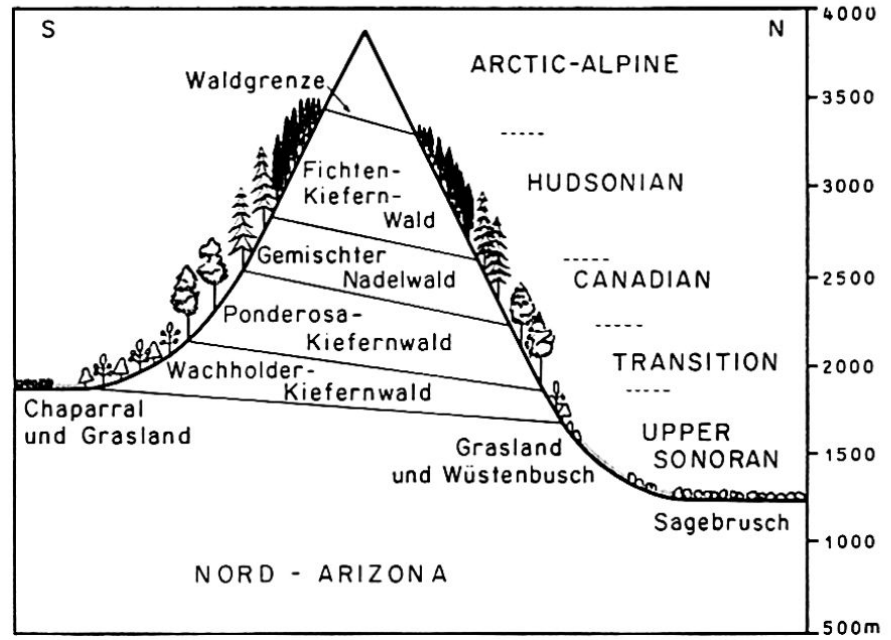

Figur 2. Vegetationsstufen («life zones») in Nordarizona in einem Idealprofil (nach Lowe 1967, p. 18). Vegetation life zones in Northern Arizona, after Lowe (1967, p. 18)

Bildung die folgenden Gesteine (Barsch and Updike, 1971): Rhyodazit, Pyroxendazit, Hypersthendazit, Andesit und Basalt. Die Reihe zeigt einen deutlichen Trend von den älteren, kieselsäurereicheren zu den jüngeren, kieselsäureärmeren Laven. In ihrer geomorphologischen Widerstandsfähigkeit besitzen diese Gesteine charakteristische Unterschiede.

Der Rhyodazit, das älteste der Ergußgesteine, ist ziemlich brüchig, was sich vor allem in angewittertem Zustand erkennen läßt. Bedingt durch das Kluftnetz, zerfällt er in Blöcke von weniger als $50 \mathrm{~cm}$ Durchmesser, die zudem schnell weiter zerfallen. Der Hauptbestandteil des Berges sowie ein ausgedehnter Erguß im NW bestehen aus Rhyodazit.

Die beiden anderen Dazite (Pyroxendazit und Hypersthendazit) sind später über die ausgedehnte Masse des Rhyodazit geflossen und bilden dadurch die Oberflächen der unteren Hangpartien. Sie sind häufig nur von geringer Mächtigkeit, was sich vor allem in den Canyons beobachten läßt. Für uns sind sie von Bedeutung dadurch, daß sie eine sehr kompakte, dichte und feinkristalline Struktur besitzen und der chemischen und physikalischen Verwitterung mit Erfolg zu trotzen vermögen. Dazu kommt, $\mathrm{da} ß$ sie über ein sehr weitmaschiges Kluftnetz verfügen, das die Bildung von großen Blöcken (über $3 \mathrm{~m}$ Durchmesser!) erlaubt.

Der Andesit tritt dagegen nur an wenigen Stellen, besonders an den oberen Hangteilen, auf. Aus ihm besteht vor allem die Gipfelpyramide des Kendrick Peak, die größeren Flächen über dem unterliegenden Rhyodazit direkt aufgesetzt ist. Der Andesit ist das kompakteste und dichteste Gestein im Untersuchungsgebiet. Er besitzt die größte Widerstands- 


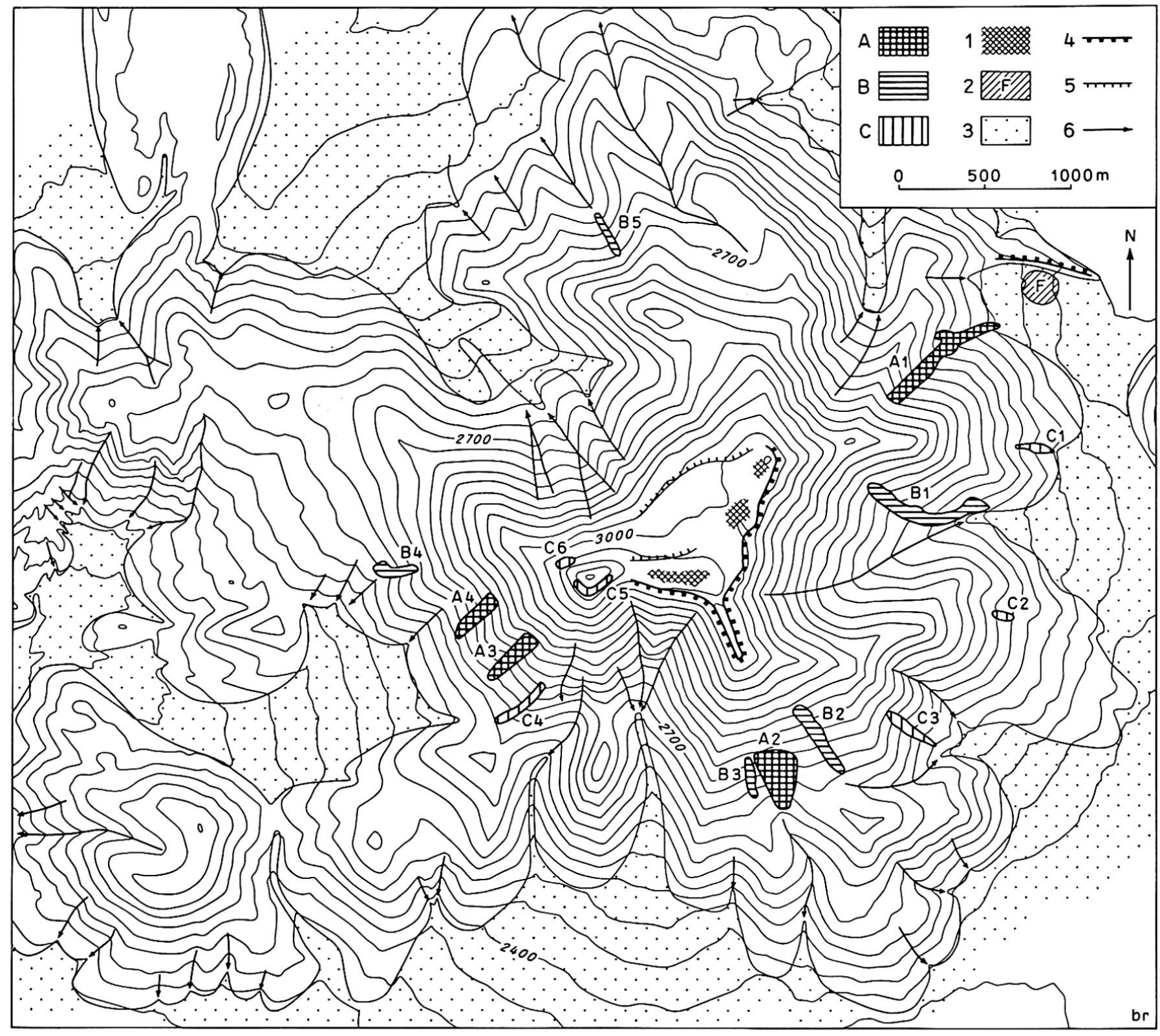

Figur 3. Der letztkaltzeitliche, periglaziale Formenschatz am Kendrick Peak in Nord-Arizona. Die 3000m-Höhenlinie entspricht der 10000 -ft-Isohypse; einVergleich mit den Originalkarten (Kendrick Peak Quadrangle und Moritz Ridge Qu., beide 1:24 000) ist dadurch leicht gewährleistet. Die Äquidistanz beträgt $30 \mathrm{~m}(100 \mathrm{ft})$. A inaktive Blockgletscher mit gut ausgeprägtem Oberflächenrelief; B inaktive Blockgletscher mit mäßig entwickeltem Oberflächenrelief; C Blockströme, Blockmeere; 1 Altiplanationsterrassen (?); 2 anstehender Fels; 3 Schwemmkegel; 4 scharf ausgeprägte Geländekante; 5 schlecht ausgeprägte Geländekante; 6 Rinne. Late Pleistocene periglacial forms at Kendrick Peak, Northern Arizona. The $3000 \mathrm{~m}$ contourline equals $10000 \mathrm{ft}$. A inactive rock glacier with well developed surface relief; B inactive rock glacier with poorly developed surface relief; C blockfield; 1 altiplanation terraces (?); 2 bedrock protruding trough fan; 3 fan; 4 well developped edge of a flat surface; 5 rounded edge; 6 furrow

fähigkeit gegen chemische Verwitterung. Aufgrund seines Kluftnetzes zerfällt er allerdings in etwas kleinere Blöcke als der Pyroxendazit und der Hypersthendazit. Häufig finden sich sogar plattenförmige Absonderungen (Dicke: etwa $10 \mathrm{~cm}$; Durchmesser: bis zu $1 \mathrm{~m})$.

Der Basalt entstammt den letzten Ergüssen aus dem Vulkan des Kendrick Peak. Es handelt sich um Olivinbasalt von mittlerer morphologischer Widerstandsfähigkeit und Neigung zu blockigem Zerfall.

\section{Untersuchungsergebnisse}

Bei unseren Untersuchungen kam es uns zunächst darauf an, die besonders typischen periglazialen Formen im Berggebiet des Kendrick Peak zu erfassen und in ihrer Verbreitung festzuhalten. Damit sollten erste Hinweise auf die Art und die Intensität der wisconsinzeitlichen periglazialen Formung gewonnen werden. In zweiter Linie wurden die Untergrenzen der verschiedenen Periglazialformen möglichst exakt in verschiedenen Expositionen be- 
stimmt. Wie zu erwarten ist, treten die periglazialen Erscheinungen am Kendrick Peak in sehr unterschiedlicher Ausprägung auf. Als auffallendste Erscheinung haben wohl die großen Blockfelder zu gelten. Dazu finden sich jedoch noch zahlreiche weitere Formen, die ihre Existenz intensiven periglazialen Vorgängen verdanken. Eine zeichnerische Zusammenfassung unserer Ergebnisse findet sich in Figur 3.

\section{Blockfelder}

\subsection{Beschreibung}

Schon mit bloßem Auge erkennt man auf der Anfahrt zum Berggebiet des Kendrick Peak zahlreiche kahle Stellen im Waldkleid der höheren Hänge. Eine Erkundung vom Flugzeug aus ergibt, da $\beta$ es sich bei diesen waldfreien Stellen in vielen Fällen nicht um sogenannte "parks", sondern um Blockwerk handelt, auf dem die Bäume nicht Fuß fassen können. Eine Ausnahme bildet nur das Blockfeld C-4, (vgl. Tab. 2), das leicht mit Vegetation bedeckt ist. Wir haben diese auffälligen Blockfelder (im ganzen 15) näher untersucht und kartiert (vgl.
Tab. 2 und Fig. 3). Dabei hat sich ergeben, daß sie alle aus groben Blöcken bestehen und das an ihrer Oberfläche feinkörniges Material vollkommen fehlt. In bezug auf das Oberflächenrelief sind sie jedoch völlig unterschiedlich gestaltet. Die einen zeigen wohlausgebildete Wälle und Rinnen mit steilen Stirnhängen (bis zu $38^{\circ}$ ), die anderen sind völlig flach und gleichförmig; schließlich gibt es noch eine weitere Gruppe von Blockfeldern, die zwischen den beiden Extremen eine vermittelnde Stellung einnimmt.

Die Blockfelder der Gruppe 1 (vgl. Tab. 2) sind alle Vollformen und durch eine deutliche Böschung vom umgebenden Gelände abgegrenzt. Diese Böschung ist im allgemeinen recht steil, zeigt aber Versturzerscheinungen: So sind in den meisten Fällen die Blöcke von der ehemaligen Oberkante nach unten gerutscht. Die Oberfläche dieser Blockfelder (vgl. Fig. 4 bis 6) ist durch Wälle und Rinnen deutlich gegliedert. Die Wälle und Rinnen laufen im oberen Teil häufig parallel zu der Richtung des Gefälles, im unteren quer dazu. Die Querwälle sind außerdem nach unten (in Richtung der Hangneigung) durchgebogen und erzeugen so ein Bild, das dem Bewegungsbild zähflüssiger Massen ähnelt. Häufig umschließen sie größere und kleinere Depressionen, in deren Umgebung die Lagerung des

Tabelle 2. Die Blockfelder am Kendrick Peak in Nord-Arizona (vgl. Fig. 3)

\begin{tabular}{|c|c|c|c|c|c|c|c|c|c|c|}
\hline \multirow[b]{2}{*}{ Name } & & \multirow[b]{2}{*}{$\begin{array}{l}\bar{\delta} \\
\text { हे } \\
\text { ळ }\end{array}$} & \multirow[b]{2}{*}{ 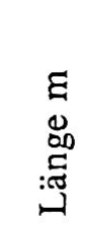 } & \multirow[b]{2}{*}{ 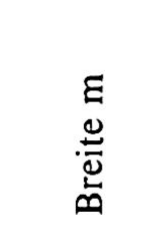 } & \multirow[b]{2}{*}{ 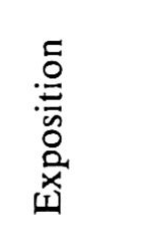 } & \multicolumn{2}{|c|}{ Höhenlage } & \multirow{2}{*}{ 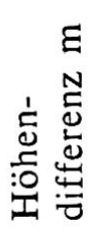 } & \multicolumn{2}{|c|}{ Gefälle } \\
\hline & & & & & & 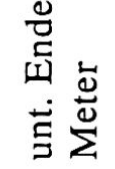 & 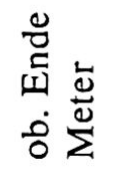 & & ஃ & $\circ$ \\
\hline \multirow[t]{4}{*}{ Gruppe 1} & Kendrick Tank Blockgletscher & A 1 & 900 & $70-75$ & $\mathrm{NE}$ & 2730 & 2485 & 245 & 23 & 10 \\
\hline & Crowley Tank Blockgletscher & A 2 & 360 & $60-240$ & $S$ & 2760 & 2615 & 145 & 40 & 18 \\
\hline & Newman Hill. Blockgletscher & A 3 & 360 & $50-100$ & SW & 2850 & 2690 & 160 & 45 & 20 \\
\hline & Newman Spring Blockgletscher & A 4 & 325 & $50-85$ & SW & 2820 & 2670 & 150 & 46 & 21 \\
\hline \multirow[t]{5}{*}{ Gruppe 2} & & B $1 *$ & 460 & 80 & S-SE & 2750 & 2610 & 140 & 30 & 14 \\
\hline & & B 2 & 480 & $50-80$ & SE & 2835 & 2670 & 265 & 55 & 25 \\
\hline & & B 3 & 240 & 50 & $\mathrm{~S}$ & 2760 & 2655 & 105 & 42 & 19 \\
\hline & & B 4 & 260 & 60 & W & 2695 & 2595 & 95 & 37 & 17 \\
\hline & & B 5 & 290 & 50 & NW & 2670 & 2565 & 105 & 36 & 16 \\
\hline \multirow[t]{6}{*}{ Gruppe 3} & & C 1 & 240 & $25-50$ & $\mathrm{E}$ & 2555 & 2485 & 70 & 29 & 13 \\
\hline & & C 2 & 145 & $35-40$ & $\mathrm{E}$ & 2630 & 2580 & 50 & 35 & 16 \\
\hline & & C 3 & 335 & $25-60$ & SE & 2700 & 2550 & 150 & 45 & 20 \\
\hline & & C 4 & 360 & $30-50$ & SW & 2770 & 2640 & 130 & 36 & 16 \\
\hline & & C $5^{* *}$ & 50 & 265 & SW_SE & 3090 & 3060 & 30 & 60 & 27 \\
\hline & & C $6 * *$ & 60 & 120 & NW & 3060 & 3010 & 50 & 83 & 37 \\
\hline
\end{tabular}

* B 1 besteht aus verschiedenen Loben, die sich aus dem Blockschutt an der Südseite des Tales entwickelt haben. Die hier zitierten Werte entsprechen dem obe ren Teil von B 1.

** C 5 und C 6 sind kleine Blockmeere an der Gipfelpyramide des Kendrick Peak. Daraus erklären sich ihre spezielle Form und die besonderen Werte für Länge und Breite. 


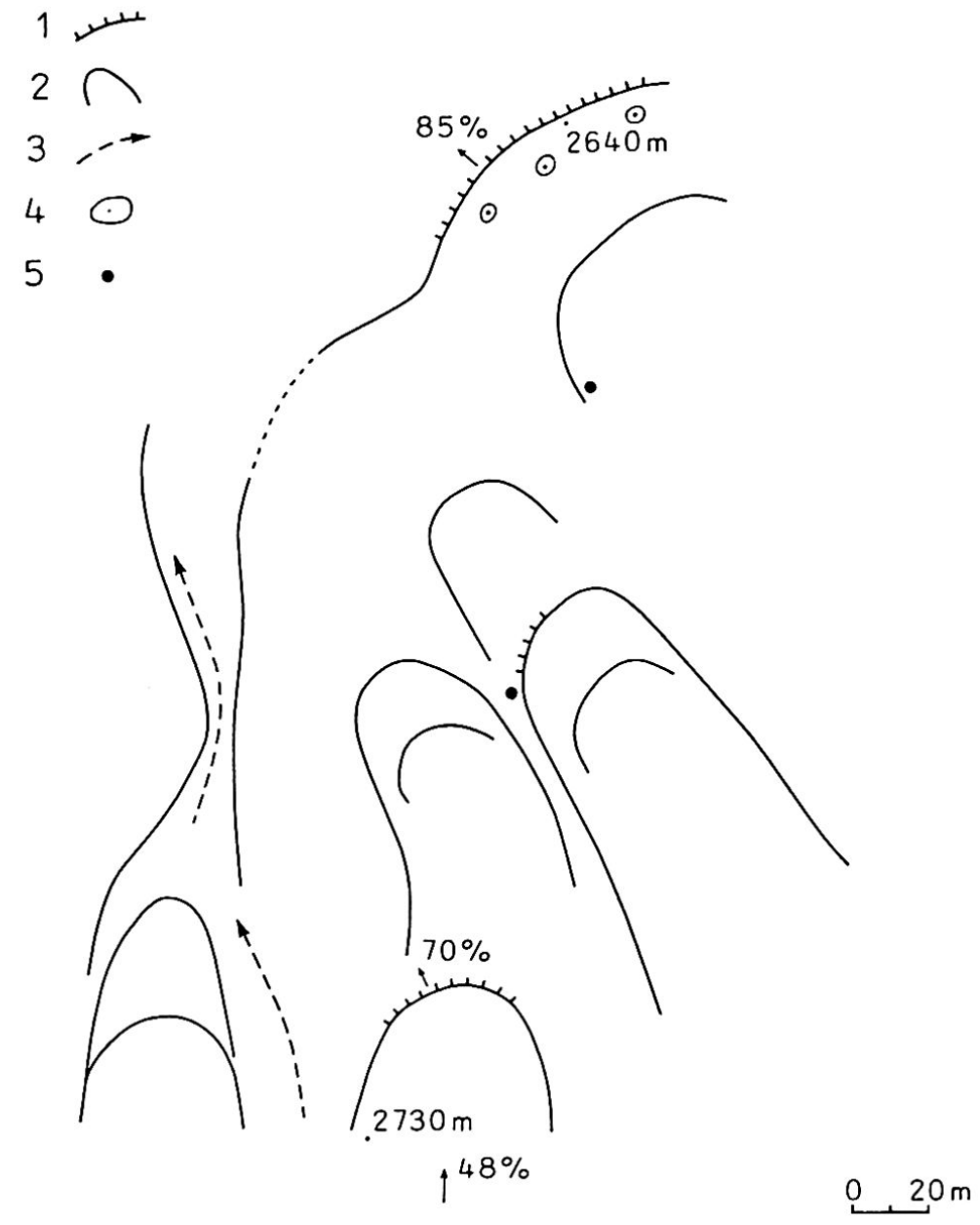

blockiger Hangschutt

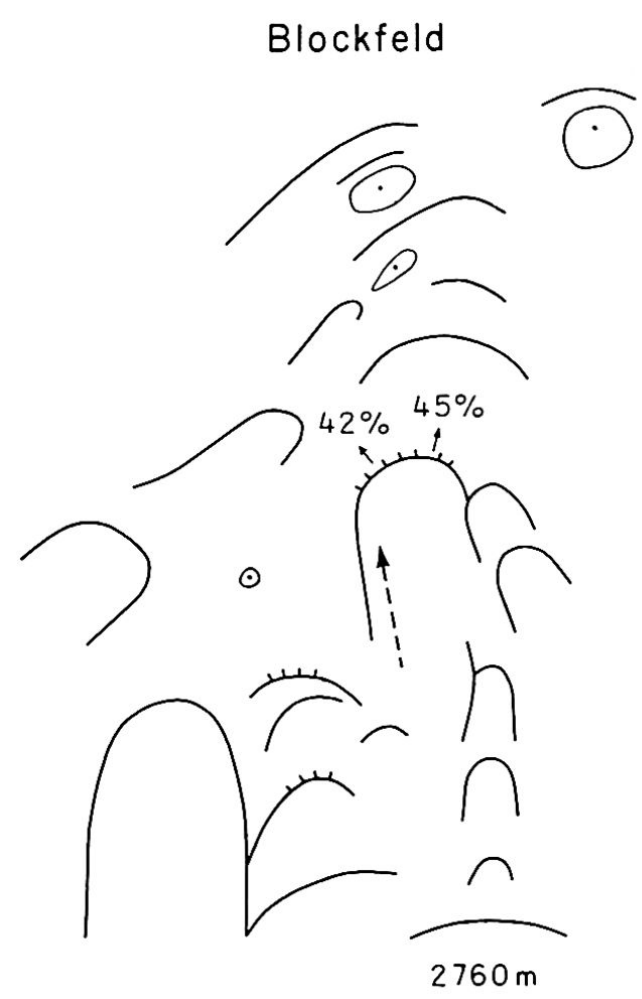

blockiger Hangschutt

Figur 4. Das Oberflächenrelief des Kendrick-Tank-(A 1, links) und Crowley-Tank-(A 2, rechts) Blockgletschers (Kendrick Peak, Nordarizona). 1 steiler Stirnhang; 2 Rücken, lobusförmiger Wall; 3 Rinne; 4 geschlossene Depression; 5 Baum. Surface relief of Kendrick Tank (A 1, left) and Crowley Tank (A 2, right) rock glacier. 1 steep slope; 2 ridge, lobe; 3 furrow; 4 closed depression; 5 tree

Blockwerks Kollapsstrukturen bzw. Versturzerscheinungen vermuten läßt. Irgendwelche sonstige Anordnungen der Blöcke an der Oberfläche der Blockfelder sind nicht zu erkennen. Die Mächtigkeit der blockigen Ablagerungen dürfte weit mehr als 10 oder $20 \mathrm{~m}$ betragen, sie ist im allgemeinen jedoch schlecht abzuschätzen, da diese Blockgebilde häufig in Tälern auftreten, welche vollständig verfüllt wurden.

Im Gegensatz dazu zeigen die Blockfelder der Gruppe 2, obwohl es sich bei ihnen ebenfalls um Vollformen handelt, ein weniger deutliches Relief. Vor allem fehlen die steilen Stirnhänge. Die geschlossenen Depressionen sind zwar vorhanden, aber flacher. Ein instruktives Beispiel bietet das Blockfeld B-1. Es hat sich unter der steilen Nordflanke eines kleinen Tales entwickelt. Die einzelnen, schwachentwickelten Loben berühren sich, und die ganze Blockmasse bedeckt fast den gesamten Talboden. Der Unterschied im Oberflächenrelief zu den Blockfeldern der Gruppe 1 ist zwar markant und klar, aber doch im wesentlichen graduell. Auf den Blockfeldern der Gruppe 2 hat wohl nie ein gut entwickeltes Relief existiert, da sie entweder relativ klein sind oder in Gebieten auftreten, in denen die Blockproduktion gering gewesen ist.

Ganz klar sind von den bisher besprochenen Blockfeldern jene der Gruppe 3 zu unterscheiden. Sie weisen überhaupt kein Relief auf; sie erscheinen trotz der rauhen Oberfläche als völlig glatt. Bruchlos gehen sie in die seitlichen Hänge über, ja in vielen Fällen erscheinen sie nicht als Vollformen, sondern als in ihre Umgebung eingesenkte Blockfelder.

\subsection{Verbreitung}

Die beschriebenen Blockfelder treten (vgl. Fig. 3) auf allen Seiten von Kendrick Peak auf. Allerdings scheint die direkte Nordexposition etwas weniger bevorzugt zu sein; eine Beobachtung, die auch in einzelnen Tälern (bei homogenem Gestein) bestätigt werden konnte: Dort findet sich unter südexponierten Hängen bedeutend mehr Schutt und demzufolge auch häufiger ein Blockfeld. Andererseits zeigt sich (Tab. 3), daß an südexponierten Hängen die 


\section{Blockfeld}

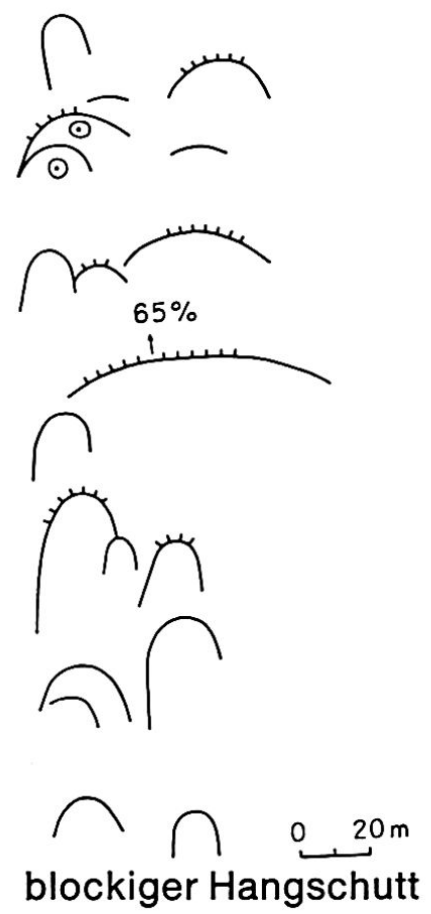

Figur 5. Das Oberflächenrelief des NewmanSpring-Blockgletschers (A 4) (Kendrick Peak, Nordarizona). Legende vgl. Fig. 4. Surface relief of Newman Spring rock glacier (A 4). Legend see Fig. 4

Untergrenze der verschiedenen Blockfeldergruppen bedeutend höher liegt als an nordexponierten. Unser statistisch gesehen allerdings noch recht kleines Material läßt einen generellen Höhenunterschied zwischen Nord und Süd von rund $200 \mathrm{~m}$ erkennen. Bei genauerer Auswertung der Tabelle 3 (vgl. auch Fig. 3) ergibt sich, daß die Blockfelder der Gruppe 1 und 2 ungefähr denselben Höhenbereich umfassen, die Blockfelder der Gruppe 3 dagegen deutlich tiefer liegen. Zwei Ausnahmen, die beiden kleinen Blockmeere C-5 und C- 6 im Bereich des äußerst widerstandsfähigen Gesteins der Gipfelpyramide (Andesit), bestätigen diese Regel.

\subsection{Genetische Deutung}

Die Formbeschreibung wie auch die beigegebenen Abbildungen der Blockfelder der Gruppe1 entspre-

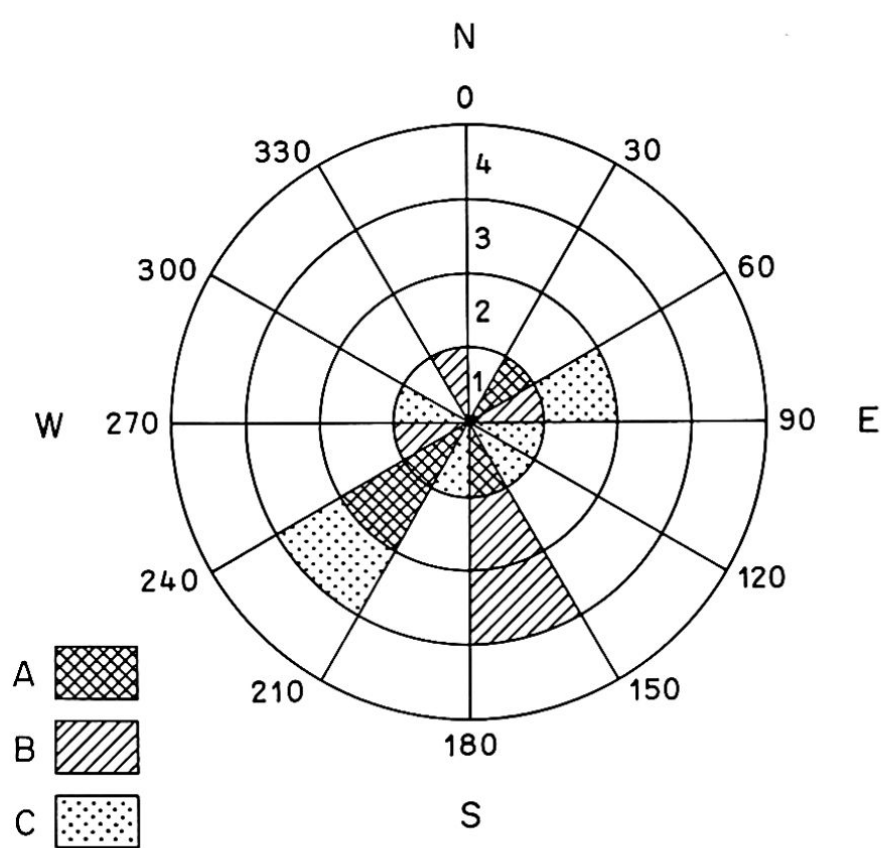

Figur 6. Exposition der Blockfelder am Kendrick Peak, (Nordarizona). A inaktive Blockgletscher mit gut ausgeprägtem Oberflächenrelief; B inaktive Blockgletscher mit mäßig entwickeltem Oberflächenrelief; C Blockstrom, Blockmeer. Orientation of boulder fields at Kendrick Peak, Northern Arizona

chen jenen von Blockgletschern, die im aktiven $\mathrm{Zu}-$ stand aus den Alpen von Barsch (1969a), aus der Colorado Front Range von White (1970) oder aus der Alaska Range von Wahrhaftig and Cox (1959) beschrieben wurden. Die Blockgletscher (rock glacier) am Kendrick Peak sind heute inaktiv, dafür sprechen nicht nur das Fehlen jeglichen Anzeichens einer Bewegung, die verstürzten Stirnhänge, die relativ feste Lagerung der Blöcke, sondern auch die Depressionen mit ihren Kollapserscheinungen. Gerade die letzteren zeigen die Stellen an, wo das einst vorhandene, für die Fortbewegung notwendige Eis (Barsch, 1969b, Wahrhaftig and Cox, 1959) abgeschmolzen ist. Nach unserer Meinung kann es sich bei diesem Eis nicht um Gletschereis gehandelt haben, wie es besonders seit der berühmten Beobachtung von Brown ${ }^{3}$ (1925) durch verschiedene Hinweise für die rezenten (aktiven) Blockgletscher in Colorado angenommen wird (Outcalt and Benedict,

Tabelle 3. Die Höhenlage $(m)$ des unteren Endes der kartierten Blockfelder nach Exposition am Kendrick Peak, Nord-Arizona Durchschnitt

Gruppe 1

Gruppe 2

Gruppe 3

$\begin{array}{cccccccc}\text { E } & \text { NE } & \text { NW } & \text { W } & \text { SW } & \text { S } & \text { SE } & \text { SW + S + SE } \\ - & 2485 & - & - & 2680 * & 2615 & - & 2658 \\ - & - & 2565 & 2595 & - & 2655 & 2670 & 2663 \\ 2483 * & - & - & - & 2640 & - & 2550 & 2595\end{array}$

* Durchschnitt von zwei Werten 
1965. White, 1970). In einem Gebiet, wo es in der letzten Eiszeit, die für die Bildung unserer Blockgletscher wohl entscheidend war, keine Gletscher gegeben hat, wird man die Blockgletscher nicht als "verschüttete Gletscher», sondern als Erscheinung des Permafrostes deuten müssen, was auch von Blagbrough and Farkas (1968) für die San Mateo Mountains in New Mexico bestätigt wird. Das Eis in ihrem Innern ist dann nicht Gletschereis, sondern aus Schnee und Schneeschmelzwasser zwischen den Blöcken neu entstanden (interstitial ice). Selbstverständlich wird der Gehalt an Eis (interstitial ice) recht unterschiedlich gewesen sein. So zeigen die meisten Blockgletscher am Kendrick Peak (Gruppe 1 der Tabelle 2) nur kleinere Depressionen, der Newman-Hill-Blockgletscher besteht dagegen in seinem unteren Ende aus einem lobusförmigen, 40 bis $50 \mathrm{~m}$ hohen Wall, der eine riesige, $10-15 \mathrm{~m}$ tiefe Depression umschließt. Offensichtlich war hier das Schutt-Eis-Verhältnis stark zu Gunsten des Eises verschobeñ.

Die Blockgletscher der Gruppe 2, die sich von denen der Gruppe 1 nur durch ein schwächeres Oberflächenrelief unterscheiden, werden entsprechend als noch unvollkommene Blockgletscher gedeutet. Ihre Unvollkommenheit beruht im wesentlichen darauf, daß sie an Stellen entstanden sind, an denen die Blockproduktion nicht ausreichend gewesen ist. So dürfte der als Beispiel geschilderte Blockgletscher B-1 als Äquivalent der «Blockgletscher unter Schutthalden» (Typ 1 nach Barsch, 1969a) aufzufassen sein.

Die Blockfelder der Gruppe 3 dagegen entsprechen wohl eindeutig den Blockströmen oder Blockmeeren der deutschen Mittelgebirge (z. B. Schott, 1931; Büdel, 1937; Fezer, 1953; Rother, 1965) oder den «blockfields» der englischen Literatur (z. B. Caine, 1966). Bei ihrer Bildung dürfte vor allem das Auswaschen von Feinmaterial zwischen den Blöcken eine große Rolle gespielt haben, ein Vorgang, der von Smith (1968) als "piping" bezeichnet worden ist. Diese Blockströme stellen also nichts anderes dar, als eine durch selektive Auswaschung entstandene Anhäufung grober Blöcke. Das wird in Arizona wie auch in den deutschen Mittelgebirgen dadurch bestätigt, daß in den randlich an die Blockströme anschließenden Gebieten heute noch Solifluktionsdecken auftreten, in denen die Blöcke in Feinmaterial eingehüllt sind.

Die Blockströme bzw. die Blockmeere haben wenn überhaupt - nur eine geringe aktive Bewegung durchgeführt. Ihre Genese, ihr Habitus, ihre Mächtigkeit usw. unterscheiden sie deutlich von den Blockgletschern. Höhenmäßig liegen sie am Kendrick Peak mit einer die Regel bestätigenden Ausnahme 4 auch deutlich tiefer als die Blockgletscher. Offensichtlich sind zu ihrer Bildung nicht mehr die rigorosen klimatischen Bedingungen notwendig wie zur Bildung der Blockgletscher.

\section{Solifluktionsschutt}

Die höher gelegenen Hänge und vor allem die oberen Teile der Täler sind im Untersuchungsgebiet durch zusammenhängende, mächtige Hangschuttdecken verhüllt. Diese Schuttdecken sind korngrößenmäßig recht wechselnd zusammengesetzt. Häufig ragen aus ihnen Blöcke heraus, die wohl nur als fossile Wanderblöcke gedeutet werden können. Gelegentlich sind aus ihnen durch Ausspülung die schon beschriebenen Blockströme entstanden, mit denen sie offensichtlich genetisch eng zusammenhängen.

Aufschlüsse sind in diesen Schuttdecken recht selten, wenn man von einigen natürlichen Anrissen in Gräben oder künstlichen Anschnitten längs kleiner Pfade absieht. Einen etwas besseren Einblick kann man nur an einigen wenigen Stellen in den unteren Bereichen bekommen, in denen gelegentlich Anschnitte beim Bau eines "Jeep-trails» gemacht worden sind. Hier zeigt sich in Höhenlagen um $2500 \mathrm{~m}$, daß ein schichtungsloser, stellenweise leicht verwürgter Schutt vorliegt, der im allgemeinen viel Feinmaterial enthält. Die Längsachsen der deutlich oblongen Fragmente sind überwiegend parallel zum Hanggefälle eingeregelt.

Aus diesen Beobachtungen ergibt sich, daß es sich bei diesem Schutt im wesentlichen um periglazialen Solifluktionsschutt handelt, der etwa gleichzeitig mit den großen Blockströmen entstanden ist. $\mathrm{Er}$ dürfte also der letzten, der Wisconsin-Kaltzeit entstammen.

Leider ist es kaum möglich eine vernünftige Untergrenze der Solifluktionsdecken anzugeben. Fossile Wanderblöcke und Hangschutt konnten bis gegen $2400 \mathrm{~m}$, also bis in den oberen Bereich der reinen Ponderosa-Kiefernwälder beobachtet werden. Es ist nicht auszuschließen, daß sie auch noch weiter nach unten reichen. Wir dürfen also festhalten, daß die letztkaltzeitliche Solifluktion bis in die obersten Bereiche der heutigen Transition life zone gereicht hat.

\section{Täler}

Eine genaue Betrachtung der Figur 3 zeigt, daß alle Täler, die ein deutliches Einzugsgebiet über $2400 \mathrm{~m}$ besitzen, große Schwemmkegel aufgeschüttet haben und meist auch eine durch Akkumulationen erzeugte breite Talsohle besitzen. Typische Beispiele dafür bilden das Kendrick Tank Valley 


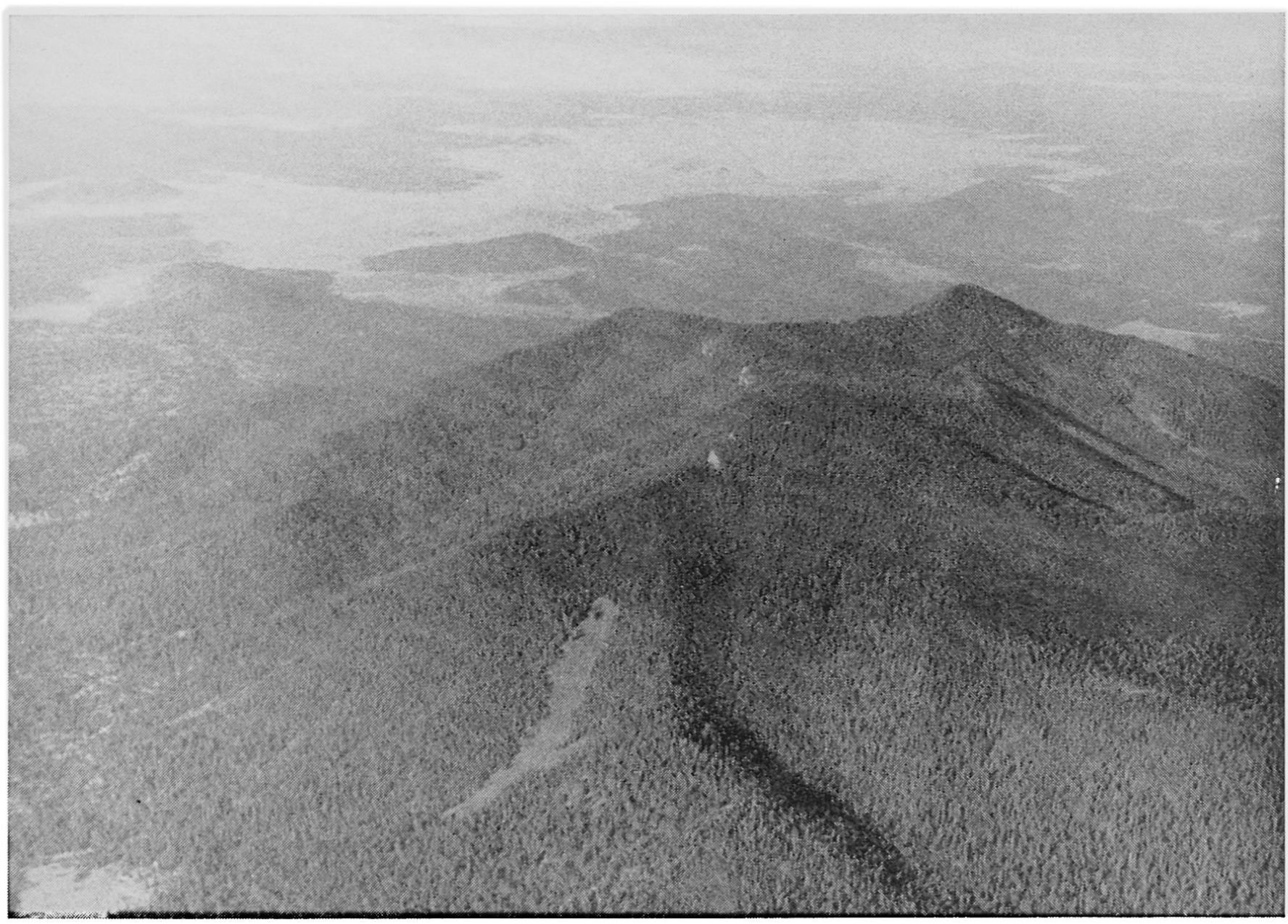

Abbildung 1. Blick von NNE aus $4000 \mathrm{~m}$ Höhe auf Kendrick Peak. Das Blockfeld im Vordergrund ist Kendrick-Tank-Blockgletscher (A 1). Die Gipfelpyramide von Kendrick Peak findet sich rechts im Bild, links davon die Schnittflächen im Rhyolit in rund $3000 \mathrm{~m}$ Höhe. Im Hintergrund die Flächen des Colorado Plateau, über die sich Kendrick Peak mit einer relativen Höhe von $900 \mathrm{~m}$ erhebt. Aufnahme 12. Juli 1969. Oblique arial photograph of Kendrick Peak (view frome NNE). The knob of Kendrick Peak is to the right. Note the flat surface cutting across the dipping bedding planes in the rhyolite (altitude about $3000 \mathrm{~m}$ ). In the background part of the Colorado Plateau towards Flagstaff. July 12, 1969

(nördlich von A 1), das Bull Basin (westlich von B 5) oder das Newman Tank Valley (westlich von A 4). Die Hänge dieser Täler sind, vor allem gegen den Talschluß, von mächtigen Schuttmassen bedeckt, die sich mit den Talbodenakkumulationen verzahnen. Stellenweise, vor allem in der Verlängerung der Talachse, sind diese Hangschuttmassen durch Gräben intensiv zerschnitten. Hangschutt und Gräben sind heute mit einem dichten Urwald bedeckt. Besondere Hinweise auf rezente aktive Schuttbildung oder gegenwärtig aktiven Abtrag konnten nicht gefunden werden. Die Täler und die Talsohlen scheinen heute nur noch sehr langsam weitergebildet $\mathrm{zu}$ werden.

Aus all dem geht hervor, daß der Hangschutt und die Talbodenakkumulationen einer morphologisch aktiveren Zeit entstammen. Da wir den Hangschutt (s. u.) als Solifluktionsschutt der letzten Kaltzeit ansehen, dürften auch die Talbodenakkumulationen und die wesentliche Ausgestaltung der heutigen Täler in die letzte Kaltzeit zu stellen sein.

\section{Schwemmkegel}

Eine weitere geomorphologisch sehr auffallende Erscheinung sind die bereits erwähnten großen Schwemmkegel, die Kendrick Peak auf fast allen Seiten umgeben. Sie beginnen teilweise weit im Berggebiet und verzahnen sich mit den Talbodenakkumulationen der Täler und Canyons, die die Hänge von Kendrick Peak zerschneiden. Wie Figur 3 zeigt, bedecken sie flächenmäßig große Gebiete. In ihnen sind also auch entsprechende Materialmengen zur Ablagerung gekommen. Dies wird durch die Zerschneidung bestätigt, die in den Tälern (z. B. W des Blockgletschers A 2) 6-7 m betragen kann, ohne daß das Anstehende erreicht wird. Materialmäßig sind im Aufbau der Schwemmkegel alle Gesteine aus dem jeweiligen Einzugsgebiet beteiligt. Auffallend ist jedoch immer wieder der große Anteil an Rhyolitfragmenten, die ihrer geringen Widerstandsfähigkeit wegen in den Blockfeldern überall fehlen. Die Sortierung des 


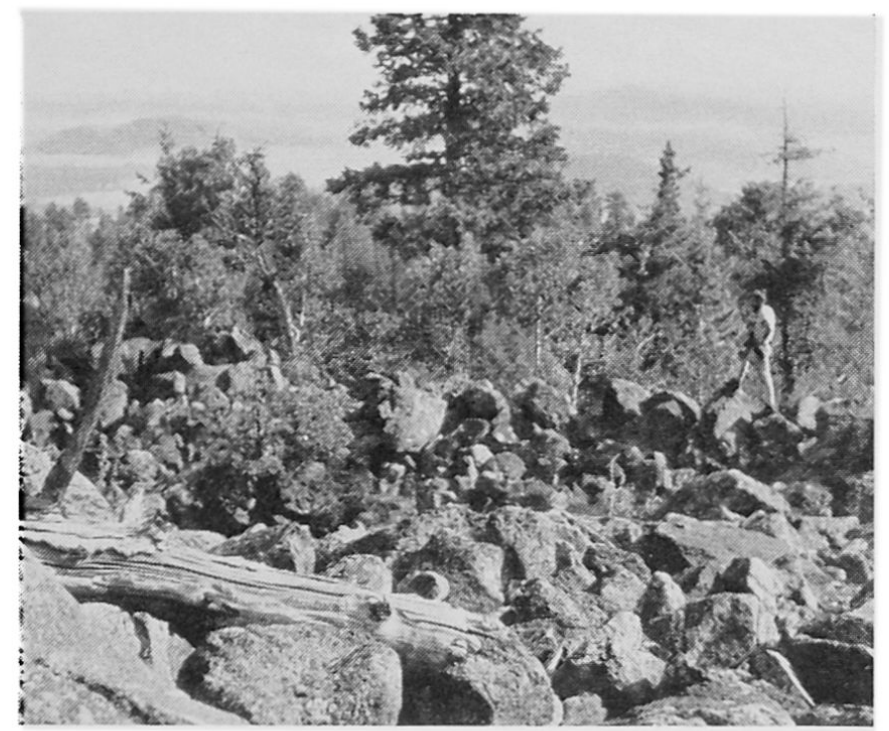

Abbildung 2. Blockwall im mittleren Teil des Newman-Spring-Blockgletschers. (A 4) Blick hangabwärts. Aufnahme 16. August 1969. Ridge on the upper part of Newman Spring rock glacier (A 4). View downslope. August 16, 1969

Materials ist schlecht. Blöcke von weit über Kubikmetergröße sind in feinen Kies oder grobe Schotter eingebettet.

Sorgsames Abgehen der einzelnen Schwemmkegel hat gezeigt, daß auf ihnen kaum Spuren rezenter Weiterbildung gefunden werden. Zwar sind hier und da Gräben (gullies) eingerissen, in denen die heutigen Hochwasser abfließen können. Doch zeigen sich an keiner Stelle jüngere, rezente Akkumulationen von einiger quantitativer Bedeutung. Wir schließen daraus, daß diese Schwemmkegel heute nicht mehr in der vorliegenden Form weitergebildet werden. Die großen Schwemmkegel des mittleren und südlichen Arizona, die allerdings bedeutend tiefer liegen, sind nach Met lon (1965) ebenfalls inaktiv. Daraus folgt, daß die Hänge im Untersuchungsgebiet heute als recht stabil anzusehen sind. Sie scheinen mit den durch die gegenwärtigen klimatischen Bedingungen gesteuerten Prozessen der Abtragung im Gleichgewicht zu stehen. Die Materiallieferung ist auch bei Hochwasser nicht groß genug, um einen weiteren schnellen Aufbau der Schwemmkegel bewirken zu können. Wir müssen also annehmen, daß zur Bildungszeit der Schwemmkegel ganz andere klimatische und geomorphologische Verhältnisse geherrscht haben als heute. Es liegt deshalb nahe, diese Schwemmkegel von Kendrick Peak mit der von Updike und Péwé (1970 a) beschriebenen Sinaguaformation in den San Francisco Mountains zu vergleichen. Es handelt sich dabei um große, schlecht sortierte Ablagerungen in Schwemmkegelform, deren Alter zwischen 2 und 0,5 Millionen Jahren liegen soll. Die Genese der Schwemmkegel ist nicht vollständig geklärt. Die

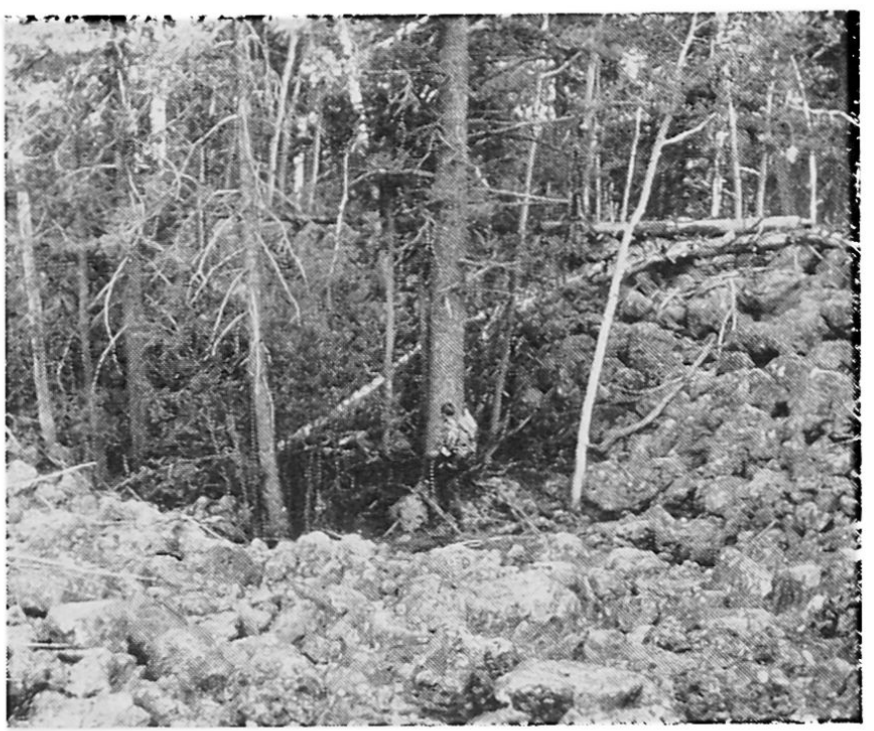

Abbildung 3. Geschlossene Depression im unteren Teil des Crowley-Tank-Blockgletschers (A 2). Aufnahme 15. August 1969. Closed depression in the lower part of Crowley Tank rock glacier (A 2). August 15, 1969

Autoren schwanken zwischen der vulkanischen (Lahars) und der periglazialen Hypothese. Die Schwemmkegel der Sinaguaformation sind heute durch Täler zerschnitten, in denen glazial-fluviale Schotter der letzten und vorletzten Eiszeit liegen. Danach müssen die Schwemmkegel am Kendrick Peak jedoch jünger sein als für die Schwemmkegel der Sinaguaformation angenommen wird, denn sonst dürfte in der letzten Kaltzeit in den Tälern am Kendrick Peak trotz periglazialer Aktivität nichts passiert sein. Das ist sehr unwahrscheinlich, da im oberen Teil der Täler und an den oberen Hängen mächtige jungpleistozäne Solifluktionsdecken anstehen, die zur Zeit ihrer Aktivität große Materialmengen den Tälern zugeführt haben. Inwieweit direkt periglaziale Solifluktion zur Ablagerung der großen Schuttmassen in und vor den Tälern beigetragen hat, läßt sich nicht mit Sicherheit entscheiden. Im Gegensatz zu den Schwemmkegeln der Sinaguaformation sind unsere Schwemmkegel aus weniger grobem Material zusammengesetzt, sie sind weniger zerschnitten, jedoch steiler und stärker konvex im Querschnitt. All das deutet darauf hin, daß die Schwemmkegel am Kendrick Peak unter weniger stürmischen, torrentiellen Verhältnissen gebildet wurden. Wie es allerdings in den tieferen, der Beobachtung nicht zugänglichen Teilen dieser Schwemmkegel aussieht, kann nicht gesagt werden. Wir nehmen an, daß die Schwemmkegel am Kendrick Peak ein letztkaltzeitliches (wisconsin-zeitliches) Alter besitzen. Zumindest muß ihre Oberfläche in der letzten Kaltzeit aktiv geformt worden sein. Es ist selbstverständlich nicht auszuschließen, daß die wisconsin-zeitlichen Schwemm- 


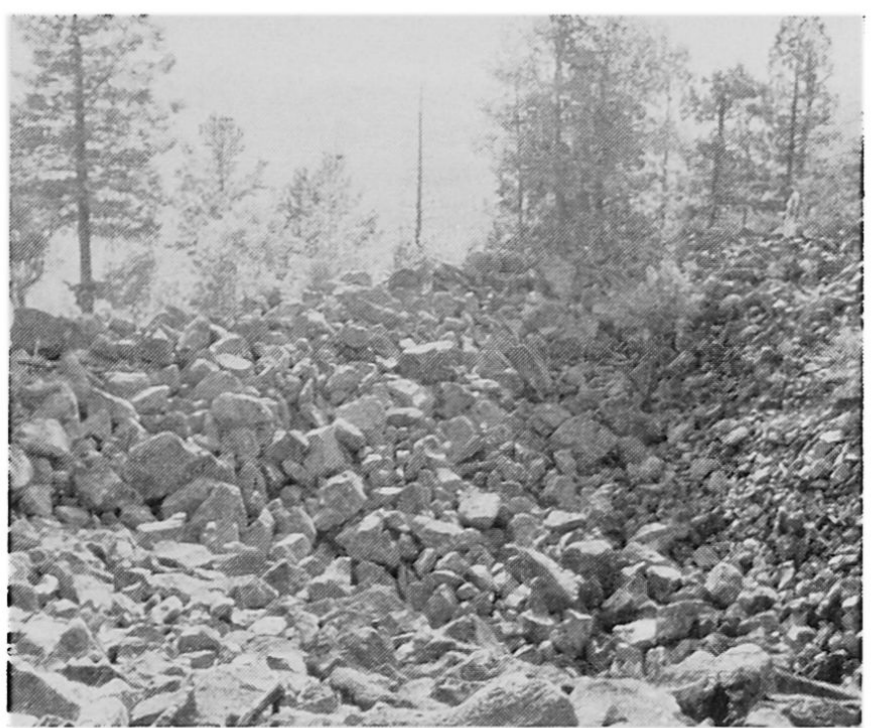

Abbildung 4. Wall und Hohlform auf dem Newman-Hill-Blockgletscher (A 3). Aufnahme 16. August 1969. Ridge and closed depression on Newman Hill rock glacier (A 3). August 16, 1969

kegel über einer der Sinaguaformation äquivalenten Ablagerung liegen. Die Deutung dieser Schwemmkegel als letztkaltzeitlich entspricht auch den Ausführungen von Melton (1965), der annimmt, daß die großen Schwemmkegel im südlichen Arizona vor allem in der vorletzten und in der letzten Kaltzeit (Illinoian und Wisconsin) gebildet worden sind.

\section{Altiplanations-Terrassen (?)}

In etwa $3000 \mathrm{~m}$ Höhe finden sich am Kendrick Peak einige größere Verflachungen, welche von der eigentlichen Gipfelpyramide aus Andesit überragt werden. Sie sind als deutliche Schnittflächen, im Rhyolit ausgebildet. Der liegende Rhyolit ist das Erstarrungsprodukt eines ehemaligen Lavastromes. Er zeigt eine ausgesprochene, an direkte Schichtung erinnernde Struktur, die sehr deutlich gegen die Horizontale geneigt ist und die von der heutigen Oberfläche glatt geschnitten wird. Im übrigen ist diese Verflachung nicht einheitlich, sondern aus verschiedenen Flächen zusammengesetzt, die durch kleinere Stufen voneinander getrennt sind. Jede dieser Flächen ist ausgesprochen eben und läßt nur ganz geringe Neigungen erkennen. Bedeckt sind sie mit sehr feinkörnigem Schutt, der im wesentlichen dem liegenden Rhyolit entstammt. Große Blöcke fehlen fast ganz. Das ist wahrscheinlich eine Folge der nicht allzu großen morphologischenWiderstandsfähigkeit des Rhyolits. Nur in der Nähe des Andesithanges der Gipfelpyramide (bis zu $70 \mathrm{~m}$ vom Hangfuß entfernt) finden sich einige größere Andesit-

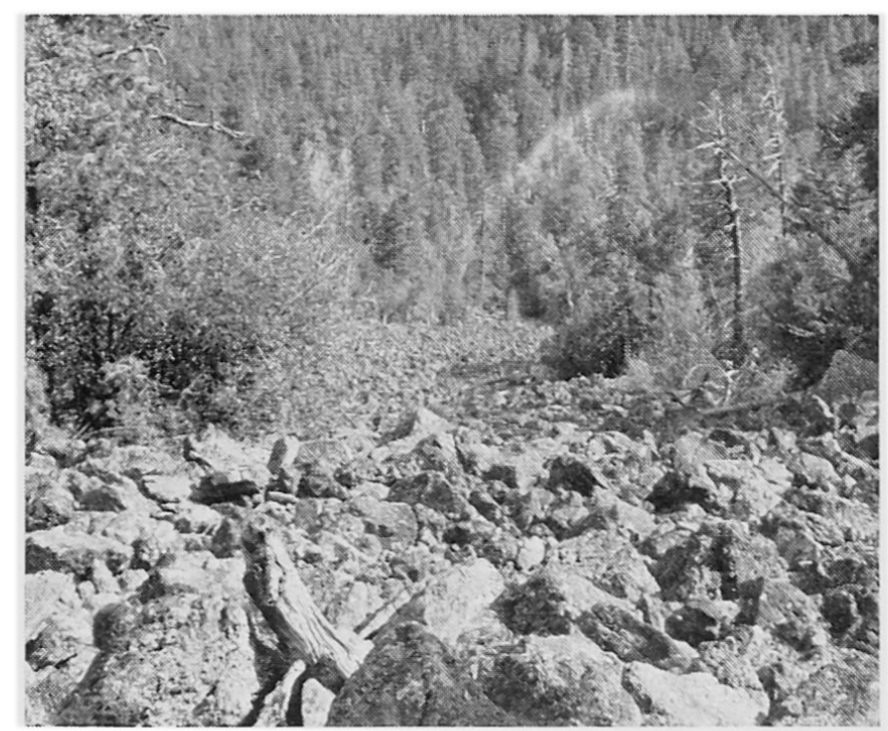

Abbildung 5. Blockstrom C 3. Blick hangabwärts. Sichtbar sind etwa $300 \mathrm{~m}$. Es ist kein Oberflächenrelief auf dem Blockstrom zu erkennen. Aufnahme 14. August 1969. Blockfield C 3. View downslope. The blockfield («rock stream») shows no surface relief. August 14, 1969

blöcke. Wahrscheinlich wurde dieser Hang im Niveau der Fläche unterschnitten, da der Übergang vom Hang zur Fläche auffallend abrupt ist. Keinesfalls bezeichnen die Blöcke jedoch die ehemalige Lage des Hangfußes, da sie durchaus beim Sturz über ein Schneefeld ihre heutige Position erreicht haben können. An den Rändern der Flächen finden sich immer wieder Relikte des anstehenden Rhyolits, die diese Fläche überragen und die man als Tors bezeichnen könnte. Die Flächen werden heute nicht mehr weitergebildet, sie sind mit dichtem Nadelwald überwachsen.

Die auffallenden Flächen sind bisher noch nicht beschrieben worden. Eine eindeutige Angabe über ihre Genese läßt sich an Ort und Stelle nicht gewinnen. Zweifellos kann es sich hier nicht um Reste alter Einebnungsflächen handeln, da Kendrick Peak ein tertiärer Vulkankegel ist, dessen Gipfel nie in eine alte Oberfläche eingebettet war. Unsere Formbeschreibung erinnert jedoch an Erscheinungen, die aus den polaren Gebieten und anderen Gebirgen als Altiplanationsterrassen (Eakin, 1916; Te Punya, 1956; Waters, 1962; Péwé, 1970) oder als Golezterrassen (Richter u. a., 1963) verschiedentlich beschrieben wurden. Die Formen vom Kendrick Peak entsprechen den Kuppen- und Hangterrassen (planated mountain crest) von Richter u. a. (1963). Strukturböden, wie sie von aktiven Altiplanationsterrassen beschrieben werden, fehlen völlig.

Die Genese der Altiplanationsterrassen wird im wesentlichen als Folge der Nivation unter der Schneegrenze (vgl. Péwé, 1970 sowie mündliche Mitteilungen von Reger und Péwé, Dept. of Geology, ASU) angesehen. Im wesentlichen dürfte die 
von Schneeflecken intensivierte Frostverwitterung sowie die dadurch bedingte seitliche Einebnung der Bergkuppe für die Bildung der Altiplanationsterrassen verantwortlich sein. Die Schneeflecken agieren in diesem Fall sowohl als Kälte- wie auch als Wasserreservoir. Von Péwé (1970) wie auch schon von Richter u. a. (1963) wird angenommen, daß die Altiplanationsterrassen nur unter einem rigorosen Periglazialklima meist dicht unter der Schneegrenze entstehen. Zahlenmäßige Angaben über das betreffende Klima fehlen jedoch noch völlig. Qualitativ läßt sich nur sagen, daß sie höhenmäßig in die Frostschuttzone der Gebirge gehören (vgl. Richter u. a., 1963).

$\mathrm{Da}$ die besprochenen Flächen von Kendrick Peak in $3000 \mathrm{~m}$ Höhe dicht unter der letztkaltzeitlichen Schneegrenze und sogar noch über der Zone der Blockgletscher gelegen haben, könnte auch für sie eine solche periglaziale Entstehung in Frage kommen. Wir neigen deshalb dazu, in diesen Flächen Altiplanationsterrassen aus der letzten Kaltzeit zu sehen, wobei vollkommen klar ist, daß eine endgültige Entscheidung erst durch weitere Untersuchungen in benachbarten Berggebieten gefällt werden kann. Diese Deutung, die unseren bisherigen Beobachtungen am besten entspricht, kann deshalb erst versuchsweise gegeben werden.

\section{Zusammenfassende Betrachtung}

Aus den bisher dargelegten Beobachtungen geht eindeutig hervor, daß die oberen Teile des Berggebietes von Kendrick Peak einem strengen Periglazialklima während der letzten Eiszeit unterlegen haben. Die Schuttbildung durch physikalische Verwitterung war in allen Teilen des Berggebietes über 2400 bis $2500 \mathrm{~m}$ sehr groß. Sie hat vor allem bei den blockbildenden Gesteinen ausgereicht, um genügend Material für die Bildung großer Blockgletscher zur Verfügung zu stellen. Die Existenz der inaktiven Blockgletscher kann als Indiz für das Klima zur Zeit ihrer Entstehung angesehen werden. Da Blockgletscher an ein Klima gebunden sind, das die Entstehung und Entwicklung von Permafrost erlaubt, muß auch in der ehemaligen Blockgletscherzone am Kendrick Peak eine negative Jahresmitteltemperatur geherrscht haben. Das bedeutet, daß die Temperaturen in dieser Höhe im Jahresmittel mindestens um 4 bis $5^{\circ} \mathrm{C}$ niedriger gewesen sein müssen als heute 5 . Auffallend ist jedoch, daß die Untergrenze der Blockgletscher am Kendrick Peak (vgl. Tabelle 4) im Mittel $700 \mathrm{~m}$ unter der eiszeitlichen Schneegrenze liegt. In den Schweizer Alpen beträgt der Abstand der rezenten Blockgletscher zur gegenwärtigen Schneegrenze nur etwa $400 \mathrm{~m}$. Man wird daraus schließen müssen, daß das Klima der letzten Kaltzeit am Kendrick Peak wie auch in den übrigen Gebirgen von Arizona einige Besonderheiten besessen hat und daß alle Übertragungen analoger Klimawerte mit Vorsicht durchzuführen sind. Wahrscheinlich dürfte der geschilderte Unterschied durch die intensivere Strahlung und die größere Aridität in Arizona verursacht sein, da beide Faktoren einen weitaus stärkeren Einfluß auf die Lage der Schneegrenze als auf die Blockgletscher haben.

Gleichzeitig mit der Bildung der Blockgletscher erfolgte eine weitgehende Verschüttung der großen Täler und der Aufbau der großen Schwemmkegel, in denen recht bedeutende Materialmengen deponiert wurden. In der Gipfelregion um 3000 m, relativ dicht unter der eiszeitlichen Schneegrenze, wurden eventuell sogar Altiplanationsterrassen gebildet, wozu nach heutiger Ansicht ein rigoroses periglaziales Klima dicht unter der Schneegrenze notwendig ist. Versuchsweise kann man daraus eine Dreiteilung der jungpleistozänen Periglazialzone zwischen der damaligen Schnee- und Waldgrenze vornehmen:

1. In der obersten Region um $3000 \mathrm{~m}$ herrschte Nivation und eventuell die Bildung von Altiplanationsternassen.

2. In der darunter anschließenden Zone bis etwa $2650 \mathrm{~m}$, die noch zur oberen subnivalen Höhenstufe gehört, wurden die großen Blockfelder gebildet. Von ihnen sind vor allem die Blockglet-

Tabelle 4. Höhendifferenz zwischen der wisconsinzeitlichen Schneegrenze von $3300 \mathrm{~m}$ und dem oberen und unteren Ende der inaktiven Blockgletscher von Typ $A$ und B am Kendrick Peak, Nord-Arizona

\begin{tabular}{|c|c|c|c|c|}
\hline \multirow[t]{13}{*}{ Blockgletscher } & $\begin{array}{l}\overrightarrow{0} \\
\text { है } \\
\text { ह }\end{array}$ & 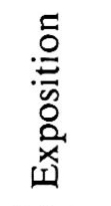 & 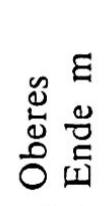 & 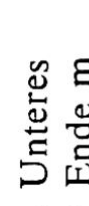 \\
\hline & A 1 & $\mathrm{NE}$ & 600 & 845 \\
\hline & A 2 & $S$ & 570 & 715 \\
\hline & A 3 & SW & 480 & 640 \\
\hline & A 4 & SW & 510 & 660 \\
\hline & \multicolumn{2}{|c|}{ Durchschnitt: } & 540 & 715 \\
\hline & B 1 & S-SE & 580 & 720 \\
\hline & B 2 & SE & 495 & 66 \\
\hline & B 3 & $S$ & 570 & 675 \\
\hline & B 4 & W & 635 & 735 \\
\hline & B 5 & NW & 660 & 76 \\
\hline & \multicolumn{2}{|c|}{ Durchschnitt: } & 508 & 71 \\
\hline & \multicolumn{2}{|c|}{$\begin{array}{l}\text { Durchschnitt } \\
\text { aller Werte: }\end{array}$} & 522 & \\
\hline
\end{tabular}


scher als Leitform dieser Höhenstufe anzusehen (Barsch, 1969 b).

3. Im untersten Teil der periglazialen Zone, hier unter $2650 \mathrm{~m}$, herrschte weitverbreitet Akkumulation in den Tälern, an den Hängen vor allem Solifluktion. Die Untergrenze dieser Höhenstufe konnte nicht genau bestimmt werden. Es wird jedoch vermutet, daß sie bei etwa $2400 \mathrm{~m}$ gelegen hat. Durch fluviale Wirkungen wurde jedoch auch unterhalb dieser Höhenlinie noch Material periglazialer Herkunft in den großen Schwemmkegeln abgelagert.

Der Vergleich mit den heute vorhandenen Vegetationszonen zeigt, daß die jungpleistozäne Waldgrenze mindestens $1000 \mathrm{~m}$ unterhalb der heutigen gelegen haben muß, die in den benachbarten San Francisco Peaks zu 3400 bis 3600 m bestimmt werden kann. Dieser Wert für die Depression der Vegetationszonen im Südwesten entspricht den Vorstellungen von Martin, der - zahlreicher Schwierigkeiten wegen - sehr zurückhaltend eine Verschiebung der Höhenzonen während des Wisconsin von 900-1200 m annimmt (z. B. Martin and Mehringer, 1965). Die Zone der inaktiven Blockgletscher entspricht etwa der mittleren Canadian life zone (gemischter Nadelwald), und die untersten Zonen periglazialer Aktivität fallen in den oberen Bereich der heutigen Transition life zone (Ponderosa-Kiefernwald). Diese Beziehungen müssen auf ihre allgemeine Gültigkeit in anderen Gebirgen des Südwestens noch weiter überprüft werden; sie lassen jedoch auch so schon einige Schlüsse auf die Verschiebung der einzelnen Höhenstufen zu. Ihre weitere Untersuchung dürfte zu einem besseren Verständnis der landschaftlichen, der klimatischen, der geomorphologischen und der pflanzengeographischen Veränderungen während des Jungpleistozäns im Südwesten der USA und darüber hinaus in den Trockengebieten der Erde führen.

\section{Zusammenfassung}

In den höheren Gebirgen des ariden und semi-ariden Südwestens der Vereinigten Staaten sind bisher kaum umfassende Studien über die letztkaltzeitliche (Wisconsin) periglaziale Höhenstufe durchgeführt worden. Wir haben deshalb in diesem Gebiet eine gezielte Untersuchung vorgenommen. Als Untersuchungsgebiet haben wir das Berggebiet von Kendrick Peak in Nordarizona $(28 \mathrm{~km}$ nördlich von Flagstaff) ausgesucht, da Kendrick Peak mit 3175 m bis diaht unter die letztkaltzeitliche Schneegrenze von $3300 \mathrm{~m}$ reicht und damit voll in der damaligen periglazialen Höhenzone gelegen hat. Heute ist der Berg, der morphologisch ein erloschener Vul- kankegel ist, völlig bewaldet (heutige regionale Waldgrenze: $3400-3600 \mathrm{~m}$ ). Spuren rezenter Periglazialerscheinungen sind nicht vorhanden.

Im Untersuchungsgebiet konnten verschiedene inaktive Periglazialformen erkannt werden, wie Blockfelder und Solifluktionsdecken, Schwemmkegel und Talverfüllungen aufgebaut aus Frostschutt sowie kleinere, die Strukturen des Liegenden schneidende Verflachungen, die möglicherweise als Altiplanationsterrassen gedeutet werden können.

Die Blockfelder bestehen aus grobem, kantigem Blockmaterial. Sie zeigen deutliche Unterschiede in ihrem Oberflächenrelief: So besitzen die als Typ A bezeichneten Blockfelder eine deutliche Gliederung in Wälle und Rinnen, die Typen B weisen nur noch ein schwach und undeutlich ausgeprägtes Relief auf und die Typen $C$ sind - trotz der großen Rauhigkeit - völlig flach. Die Typen A und B werden als heute inaktive Blockgletscher der letzten Kaltzeit gedeutet. Da Kendrick Peak nicht vergletschert war, muß das zur Bewegung dieser Blockgletscher notwendige Eis Bodeneis («interstitial ice») gewesen sein. Die Typen $C$ werden als Blockströme bzw. Blockmeere angesehen, die durch Auswaschung von Feinmaterial aus Solifluktionsdecken entstanden sind, mit denen sie sich gegen die seitlichen Hänge verzahnen. Diese Solifluktionsdecken zeigen im Untersuchungsgebiet eine weite Verbreitung. Ihre Untergrenze ist schwer festzulegen, dürfte aber bei $2400 \mathrm{~m}$ liegen. Die Solifluktionsdecken sind außerdem mit den Talschuttmassen und daher auch mit den großen Schwemmkegeln verknüpft, die das Berggebiet auf allen Seiten umgeben. Die Schwemmkegel werden heute nicht mehr weitergebildet; sie ähneln darin den Schwemmkegeln der Sinagua Formation (Updike und Péwé). Allerdings existieren auch einige Unterschiede: So sind die Schwemmkegel am Kendrick Peak nur geringfügig zerschnitten, d. h. sie müssen - zumindest an der Oberfläche - auch während der letzten Kaltzeit noch intensiv überformt worden sein, da sie mit den mächtigen Talschuttmassen weiter oberhalb verknüpft sind. Die Schwemmkegel am Kendrick Peak müssen deshalb jünger sein als die der Sinaguaformation, wo die Schotter der letzten beiden Kaltzeiten in Rinnen liegen.

In ca. $3000 \mathrm{~m}$ Höhe treten am Kendrick Peak einige breite, recht ebene und terrassenähnliche Verflachungen auf, die die Strukturen des liegenden Rhyolit deutlich schneiden. Randlich zu diesen Flächen finden sich immer wieder kleinere Aufragungen aus anstehendem Rhyolit. Offensichtlich liegt hier eine Einebnung, wahrscheinlich periglazialen Oharakters, vor. Möglicherweise können diese Verflachungen als Altiplanationsterrassen gedeutet werden, die hier durch Nivation während der letzten Kaltzeit dicht unter der damaligen Schneegrenze entstanden sind. 
Die ehemalige periglaziale Höhenzone kann am Kendrick Peak in drei Teile gegliedert werden:

1. Oberhalb $3000 \mathrm{~m} \mathrm{muß} \mathrm{die} \mathrm{periglaziale} \mathrm{Aktivität}$ äußerst intensiv gewesen sein. Nivation und wahrscheinlich Altiplanation herrschten vor.

2. Zwischen 3000 und $2650 \mathrm{~m}$ liegt die Zone der großen Blockgletscher. In ihr muß zur letzten Kaltzeit Permafrost möglich gewesen sein.

3. Unterhalb $2650 \mathrm{~m}$ bis ca. $2400 \mathrm{~m}$ überwiegt die Solifluktion, als deren Folge Solifluktionsdekken, Blockströme bzw. Blockmeere sowie die Talverfüllung anzusehen sind. Durch fluviale Prozesse wurde außerdem Material aus dieser Zone abtransportiert und in den großen Schwemmkegeln rund um Kendrick Peak zur Ablagerung gebracht.

Während der letzten Kaltzeit muß die Waldgrenze im Gebiet von Kendrick Peak mindestens $1000 \mathrm{~m}$ niedriger gelegen haben als heute; die periglaziale Höhenstufe, die heute völlig fehlt, hat damals eine Höhendifferenz von 800-900 m umfaßt, wobei die Untergrenze etwa bei $2400 \mathrm{~m}$ gelegen haben dürfte.

\section{Summary}

Periglacial landforms of Wisconsinan time on Kendrick Peak, Northern Arizona

In the higher mountains of the arid and semi-arid southwestenn United States anly a few comprehensive studies of the Wisconsinan periglacial zone have been made. In order to furnish more detailed information on the late Pleistocene periglacial landforms we selected the Kendrick Peak area $(28 \mathrm{~km}$ north of Flagstaff) for closer research and mapping. The altitude of this mountain $(3,175 \mathrm{~m})$, just below the regional late Wisconsinan snowline $(3,300 \mathrm{~m}$ on the San Francisco peaks according to Updike, 1969), indicates that Kendrick Peak supported a full periglacial environment during Wisconsinan times. Today the old volcano, part of the San Franciscan Volcanic Field, is heavily forested by spruce and fir in the upper parts, and by Ponderosa pine at the lower elevations. No signs of present day periglacial phenomena have been recorded.

In the Kendrick Peak area several distinct, but inactive, periglacial phenomena can be described: boulderfields and solifluction deposits, fans and valley floor accumulation, and flat surfaces tentatively named as altiplanation terraces.

The boulderfields consist of coarse, angular blocky material. They show very distinct differences in surface relief. The type A boulderfields have a very prominent relief of furrow and ridges, the type $B$ boulderfields have a poorly developed relief, and the type $\mathrm{C}$ boulderfields show no relief. The first two groups (types A and B) are believed to be inactive rock glaciers, formed during Wisconsinan time. In an area lacking glaciation they must have moved with interstitial ice. The type $\mathrm{C}$ boulderfields are blockfields (Blockmeere, Blockströme), which have been formed by outwash and piping. Normally they interfinger with solifluction deposits on the neighboring slopes. These solifluction deposits cover nearly all slopes as far down as 2400 to $2500 \mathrm{~m}$, but their exact lower limit is difficult to establish. They are connected with the valley fill, which forms relatively flat valley floors and which is related to the material in the big fans. It seems that the valleys which have their roots above $2400 \mathrm{~m}$ produced big fans. These fans are inactive today, and they may be compared with the Sinagua formation established on the San Francisco Peaks by Updike and Péwé. But there are some differences: the fans around Kendrick Peak are only slightly dissected. They must have been formed, at least at the surface, during Wisconsinan time, because the valley fill is related to solifluction deposits and to rock glaciers of late Pleistocene age. The surface of the fans around Kendrick Peak is therefore younger than the surface of the fans of the Sinagua formation.

At an elevation of about $3000 \mathrm{~m}$ some broad flat, surfaces have been observed cutting across the dipping bedding planes of the rhyolite. Around their perimeter several knobs of the rhyolite are preserved. These surfaces must have been formed by some type of planation process. Based upon our observations we feel that the most logical explanation for the flat-topped crest of this mountain is to assume a periglacial origin. Tentatively we propose therefore that these flat surfaces are altiplanation terraces formed by nivation during Wisconsinan time just below snowline.

The former periglacial zone at Kendrick Peak can now be divided into three parts:

1. Above $3000 \mathrm{~m}$ periglacial activity must have been very important. Nivation and perhaps altiplanation occured.

2. Below $3000 \mathrm{~m}$ and down to $2650 \mathrm{~m}$ rock glaciers are the characteristic form. During their time of activity permafrost must have been common in this altitude range.

3. The lowest part, between 2650 and ca. $2400 \mathrm{~m}$ produced solifluction. Blockfield deposits and valley fill are the main features of this zone. By fluvial action part of the periglacial material has been washed out and was deposited below the periglacial zone in the fans around Kendrick Peak. 
Timberline must have been clearly, at least $1000 \mathrm{~m}$, depressed during late Pleistocene time. The periglacial zone of this time had a difference in elevation of about 800 to $900 \mathrm{~m}$, its lower limit being about $2400 \mathrm{~m}$.

\section{Anmerkungen}

1 Die Kartierungen und Untersuchungen für die vorliegende Studie wurden im Jahr 1969 durchgeführt. Während dieser Zeit hat sich $\mathrm{D}$. Barsch mit Hilfe des Schweizerischen Nationalfonds (Bern) und des Fonds zur Förderung von Lehre und Forschung (Basel) an der Arizona State University aufgehalten; R. Updike hat zu dieser Zeit im Rahmen eines Forschungsprogramms des Department of Geology der Arizona State University quartärgeologische Untersuchungen in den San Francisco Mountains durchgeführt. Beide Autoren sind Troy L. Péwé, Chester F. Royse jr., und Michael F. Sheridan (alle vom Department of Geology an der Arizona State University) für ihre dauernde Diskussionsbereitschaft zu großem Dank verpflichtet. Die freundschaftliche Kritik der genannten Herren hat uns sehr geholfen; für eventuelle Irrtümer bleiben wir jedoch allein verantwortlich. An dieser Stelle möchten wir auch den Institutionen, wie dem Schweizerischen Nationalfonds (Bern), dem Fonds zur Förderung von Lehre und Forschung (Basel) und dem Museum of Northern Arizona (Flagstaff), danken, von denen wir unabhängig voneinander unterstützt worden sind.

2 Ausnahmen von dieser Regel bilden etwa die Arbeiten von Hunt (1956) oder Hunt u. a. (1953), die wie auch Richmond (1962) oder Flint and Denny (1958) Hinweise auf letztkaltzeitliche periglaziale Ablagerungen geben. In all diesen Fällen werden jedoch Gebiete bearbeitet, die viel weiter nördlich liegen als die hier behandelten. Erst in den letzten Jahren ist vor allem von Blagbrough mit der Bearbeitung von letztkaltzeitlichen periglazialen Formen im Südwesten, besonders in New Mexico, begonnen worden (Blagbrough and Bread, 1967; Blagbrough and Farkas, 1968).

3 Brown (1925) beschreibt aus den San Juan Mountains in Südost-Colorado einen Tunnel, der längs durch einen Blockgletscher ins anstehende Gestein vorgetrieben wurde. Nach seinen Angaben erschloß der Tunnel zunächst 6-8 m grobblockigen Schutt, dann rund $100 \mathrm{~m}$ gefrorenen Schutt und schließlich rund $30 \mathrm{~m}$ Eis (clean ice), bevor das Anstehende erreicht wurde. Dabei ist zwar nicht entschieden, daß es sich um echtes Gletschereis handelt, doch liegt die Vermutung nahe.
4 Die Ausnahme bilden die beiden Blockmeere C5 und $\mathrm{C} 6$ an der Gipfelpyramide des Kendrick Peak (vgl. Tabelle 2). Petrographisch bestehen sie aus dem sehr widerstandsfähigen Andesit.

5 Dieser Wert ist nur als Minimum zu verstehen. Die von Galloway (1970) angenommene Erniedrigung der mittleren Temperaturen von $10-11^{\circ} \mathrm{C}$ für den Südwesten der USA scheint uns allerdings als zu hoch.

\section{Literatur}

Atwood W. W. (1905): Glaciation of the San Francisco Mountain. Arizona. Jour. Geology 13, 276 279.

Barsch D. (1969 a): Studien und Messungen an Blockgletschern in Macun, Unterengadin. Zeitschrift f. Geomorphologie, Supplementband 8, 1130.

- (1969 b): Permafrost in der oberen subnivalen Stufe der Alpen. Geographica Helvetica 24, 10-12. - and Updike R. G. (1971, in press): Late Pleistocene periglacial geology (rock glaciers and blockfields) at Kendrick Peak, Northern Arizona. Arizona Geol. Soc. Digest.

Blagbrough J. W., Bread W. J. (1967): Protalus ramparts on Navajo Mountain, Southern Utah. Am. Jour. Sci. 265, 759-772.

— and Farkas S. E. (1968): Rock glaciers in the San Mateo Mountains, South-Central New Mexico. Am. Jour. Sci. 266, 812-823.

Brown W. H. (1925): A probable fossil glacier. Journal of Geology 33, 464-466.

Büdel J. (1937): Eiszeitliche und rezente Verwitterung und Abtragung im ehemals nicht vereisten Mitteleuropa. Petermanns Mitteilungen, Erg. H. 229, $71 \mathrm{p}$.

Caine N. (1966): The Blockfields of Northeastern Tasmania. Australian National University, Canberra, Dept. of Geography, Publ. G 16, 127 p.

Colton H. S. (1958): Precipitation about the San Francisco Peaks, Arizona. Museum of Northern Arizona Tech. series, No. 2, 18 p.

- (1967): The basaltic cinder cones and lava flows of the San Francisco Mountain vulcanic field.

Museum of Northern Arizona, Flagstaff. 58 p.

Fezer F. (1953): Schuttmassen, Blockdecken und Talformen im nördlichen Schwarzwald. Göttinger Geogr. Abhandlungen 14, 45-77.

Flint R. F., Denny C. S. (1958): Quaternary geology of Boulder Mountain, Aquarius Plateau, Utah. U. S. Geol. Surv. Prof. Bull. 1061-D, 103-164.

Galloway R. W. (1970): The full glacial climate in the southwestern United States. Annals Assoc. Amer. Geographers 60, 245-256. 
Green C. R., Sellers W. D. (1964): Arizona climate. The Univ. of Arizona Press, Tucson, Ariz., 503 p. Hunt C. B., Averitt P., Miller R. L. (1953): Geology and geography of the Henry Mountains region, Utah. U. S. Geol. Surv. Prof. Paper 228, 234 p.

Hunt C. B. (1956): Cenozoic geology of the Colorado Plateau. U. S. Geol. Surv. Prof. Paper 279, 99 p.

Lowe C. H. (1967): The vertebrates of Arizona. The University of Arizona Press, Tucson, 270 p.

Martin P. S., Mehringer Jr. P. J. (1965): Pleistocene pollen analysis and biogeography of the Southwest. In: Wright Jr., H. E. and Frey, D. G.: The Quaternary of the United States: Princeton University Press, 433-451.

Melton M. A. (1965): The geomorphic and paleoclimatic signifiance of alluvial deposits in southern Arizona. Journal of Geology 73, 1-38.

Merriam C. H. (1890): Results of a biological survey of the San Francisco Mountains region and desert of the Little Colorado in Arizona. U.S. Dept. Agr., North Amer. Fauna No., 3, 136 p.

Outcalt S. J., Benedict J. B. (1965): Photo-interpretation of two types of rock glaciers in the Colorado Front Range, U.S.A. Jour. Glaciology 5, 849-856. Péwé T. L. (1970): Altiplanation terraces of early Quarternary age near Fairbanks, Alaska. Acta Geographica Lodziensia 24, 357-363.

Richmond G. M. (1962): Quaternary stratigraphy of the La Sal Mountains, Utah. U.S. Geol. Surv. Prof. Paper 324, 135 p.

Robinson H. H. (1913): The San Francisco volca- nic field, Arizona. U. S. Geol. Survey Prof. Paper 76, $213 \mathrm{p}$.

Rother K. (1965): Ein Beitrag zum Blockmeerproblem. Zeitschrift für Geomorphologie 9, 321-331. Schott C. (1931): Die Blockmeere in den deutschen Mittelgebirgen. Forschungen zur deutschen Landes- und Volkskunde 29, 78 p.

Sharp R. P. (1942): Multiple Pleistocene glaciation on San Francisco Mountain, Arizona. Jour. Geology 50, 481-503.

Smith, H. T. U. (1968): "Piping» in relation to periglacial boulder concentrations. Biuletyn Periglacjalny 17, 195-204.

Troll C., Paffen K. H. (1964): Die Karte der Jahreszeiten-Klimate der Erde. Erdkunde 18, 5-28.

Updike R. G. (1967): Multiple Pleistocene glaciation of the San Francisco Peaks, Arizona (abst.). Ariz. Acad. Sci. Jour. 5, 24-25.

- (1969): Glacial geology of the San Francisco Peaks, Arizona: Arizona State University, Unpubl. Master's thesis, $154 \mathrm{p}$.

- and Péwé T. L. (1970 a): A new Quarternary formation in Northern Arizona. Plateau (Museum of Northern Arizona, Flagstaff) 43, 21-26.

- (1970 b): The glacial and related Quarternary events of the San Francisco Peaks, Arizona. aus: C. T. Smith, Guidebook to Four Corners, Colorado Plateau, Central Rocky Mountain Region, 39-42. Wahrhaftig C., Cox A. (1959): Rock glaciers in the Alaska Range. Geol. Soc. America Bull. 70, 383 435. 